\title{
Identification of Anti-Inflammatory and Anti-Hypertensive Drugs as Inhibitors of Bacterial Diguanylate Cyclases
}

\author{
Helton J. Wiggers, *,\# Edson Crusca, Éverton E. D. Silva, Juliana Cheleski, ${ }^{\#}$ \\ Naiara U. Torres and Marcos V. A. S. Navarro
}

\author{
Departamento de Física e Ciência Interdisciplinar, Instituto de Física, Universidade de São Paulo, \\ 13566-590 São Carlos-SP, Brazil
}

\begin{abstract}
Biofilms are widely present in many human chronic infections, often more resistant to treatment with antibiotics. Bacterial diguanylate cyclases (DGCs) synthesize cyclic dimeric guanosine monophosphate (c-di-GMP) from two guanosine-5' -triphosphate (GTP) molecules. c-di-GMP is a central second messenger controlling biofilm formation, turning this class of enzymes an attractive target to prevent and disrupt biofilms of pathogenic bacteria. Here, we apply an in silico ligand- and target-based hybrid method to screen potential DGC inhibitors from an FDA-approved drug databank. Mass spectrometry assays confirmed that seven screened compounds selectively bound to the GTP active site of $P$. aeruginosa WspR GGDEF domain. Four out of those, including the anti-inflammatory sulfasalazine and the anti-hypertensive eprosartan, inhibited distinct DGCs (P. aeruginosa $\mathrm{WspR}$ and E. coli $\mathrm{YdeH}$ ) in the micromolar range. Sulfasalazine and eprosartan reduced aggregation in solution of $E$. coli overexpressing WspR or YdeH. Similar anti-aggregation effects were also observed for sulfasalazine-related anti-inflammatory drugs sulfadiazine and sulfathiazole, the latter a previously described anti-biofilm agent. The optimized pharmacokinetic properties and toxicological profiles of the DGC inhibitors could be promising candidates for new anti-microbial agents based on the drug reposition strategy.
\end{abstract}

Keywords: c-di-GMP, diguanylate cyclase enzymes, bacterial biofilm, virtual screening, drug repositioning

\section{Introduction}

Bacterial biofilms, a multicellular community encased in an extracellular matrix, are commonly related to persistent infections, usually less susceptible to antibiotic treatments when compared to planktonic cells. ${ }^{1-3}$ According to the National Institute of Health, ${ }^{4}$ up to $80 \%$ of human bacterial infections involve biofilm-associated microorganisms. For instance, biofilm formation plays a crucial role in microbe pathogenicity such as Legionnaire's disease, endocarditis, pneumonia accompanied by cystic fibrosis and infections of urogenital and gastrointestinal tracts. ${ }^{5,6}$ Furthermore, biofilm infections promote devastating effects on medical device implanted and immunocompromised individuals, representing a major medical and economic predicament. $^{7}$ The burden on public health due to chronic biofilm contaminations urge for the development of new chemotherapeutic approaches. Cellular processes

*e-mail: wiggershelton@gmail.com

\#Current address: Centro de Engenharias e Ciências Exatas (CECE), Universidade do Oeste do Paraná (UNIOESTE), 85903-000 Toledo-PR, Brazil controlling biofilm formation and dispersion, such as quorum sensing systems and metabolic pathways, are important targets for the discovery of new bioactive compounds. ${ }^{8-10}$

The second messenger bis-(3'-5')-cyclic dimeric guanosine monophosphate (c-di-GMP) is a key regulator of bacterial behavior, especially controlling the switch between the motile planktonic and sedentary biofilm-associated lifestyles. Cyclic di-GMP stimulates the biosynthesis of adhesins and exopolysaccharide matrix substances in biofilms and inhibits various forms of motility. In general, low intracellular c-di-GMP levels are associated with freeswimming cells, whereas bacteria living in mature biofilms present high concentration of intracellular c-di-GMP. ${ }^{11}$ Diguanylate cyclases (DGCs) containing the GG(D/E)EF motif at the active site (A-site) synthesizes c-di-GMP from two guanosine-5' -triphosphate (GTP) molecules, while phosphodiesterases (PDEs) from the EAL and HD-GYP families break it down to 5-phosphoguanylyl-(3-5)-guanosine (pGpG) and two GMP, respectively. ${ }^{12}$ Catalytically active DGCs are usually subjected to allosteric product inhibition, 
which involves c-di-GMP binding to a secondary site (I-site) characterized by a RxxD motif. ${ }^{13-16}$ Several major human pathogens, including Pseudomonas aeruginosa, Salmonella typhimurium, and Vibrio cholerae, possess numerous c-di-GMP-metabolizing proteins, and some studies confirmed the correlation of c-di-GMP-mediated signaling with biofilm formation. ${ }^{17-19}$ Furthermore, modulation of c-di-GMP signaling by environmental changes has also been linked with the dispersion of cells from established biofilms. ${ }^{20-22}$ Therefore, drugs able to inhibit c-di-GMP synthesis are promising candidates for new anti-microbial agents with anti-biofilm activity. Indeed, genetic manipulation of c-diGMP levels through the modulation of PDE activity in $P$. aeruginosa prevented and promoted clearance of biofilm infections in mice. ${ }^{23}$

There are an increasing number of researches for bacterial DGC inhibitors, as an example, the high throughput screening of chemical libraries led to the identification of competitive DGC inhibitors able to interfere with $P$. aeruginosa and Acinetobacter baumannii biofilms. ${ }^{24,25}$ In vivo screenings showed that sulfathiazole and azathioprine have an impact on biofilm formation by indirectly decreasing c-di-GMP concentrations, probably targeting the biosynthesis of GTP. ${ }^{26,27}$ Alternatively, strategies based on c-di-GMP modification and GTP analogs also identified specific DGCs inhibitors targeting both active and the allosteric binding sites. ${ }^{28,29}$

In this report, we identified novel inhibitors of bacterial DGCs by virtual screening methods (VS) from the DrugBank database. DrugBank database has a collection of FDA-approved drugs with approximately 1500 entries that are in therapeutic use or are being tested in a wide variety of diseases. Binding assays by mass spectrometry confirmed that seven compounds selected by virtual screening interacted with the GTP active site of $P$. aeruginosa WspR GGDEF domain. The four molecules presenting highest affinity, including the anti-inflammatory sulfasalazine and the anti-hypertensive eprosartan, also inhibited purified DGCs (P. aeruginosa $\mathrm{WspR}$ and E. coli $\mathrm{YdeH}$ ) in vitro enzyme assay. Additionally, in vitro assays demonstrated the ability of some compounds to prevent bacterial aggregation, as an indication of anti-biofilm properties.

\section{Experimental}

\section{Reagents and materials}

All utilized materials were of analytical reagent grade and used as purchased, without further purification and all solutions were prepared freshly with deionized water. All reagents were purchased from Sigma-Aldrich.

\section{Computational methods}

Computational studies were carried out using the OpenEye Scientific Software, ${ }^{30}$ which develop large-scale molecular modelling applications and toolkits. Primarily aimed towards drug discovery and design. Programs package versions used in Linux workstation.

Initially, the sets of molecular structures in the sdf file format was obtained from DrugBank database,$^{31}$ then a conformer library was generated for each individual molecular structure using the OMEGA program (version 2.1.0). The algorithm implemented in OMEGA dissects molecules into fragments and reassembles them to generate many possible conformations, then submits each conformer to a simplified energy evaluation. Next, all conformers below a defined energy threshold are compared and those falling within a certain root mean square deviation (RMSD) of atomic coordinates are clustered into a single representative group. Default parameters were used, except ewindow $\left(25.0 \mathrm{kcal} \mathrm{mol}^{-1}\right)$ and maxconfs (500). Molecular charges of every conformer were calculated using QUACPAC program, with default parameters. QUACPAC attempts to make charges correctly. It includes $\mathrm{pK}_{\mathrm{a}}$ and tautomer enumeration in order to get correct protonation states, partial charges using multiple models that cover a range of speed and accuracy, and electrostatic potential map construction and storage. ${ }^{32}$ The DrugBank conformer's library was employed as input to the ligand and target based virtual screening methods.

\section{Ligand-based virtual screening methods}

ROCS (Rapid Overlay of Chemical Structures, version 2.4.1) uses a shape-based superposition method in which molecules are aligned with maximizing the overlap volume between a reference structure (the query molecule) and every conformer of the molecules contained in the database. The ligand GTP- $\alpha-S$ was used as query by the ROCS program, the structure was extracted from an X-ray structure deposited in the PDB database with ID 2 V0N in co-crystallized conformation into a diguanylate enzyme active site, therefore considered as active conformation. The degree of structural similarity was calculated using ComboScore, the sum of the Shape Tanimoto and the Scaled Color values. ${ }^{33}$

$\mathrm{EON}^{34,35}$ is an electrostatics comparison program, it compares electrostatic potential maps of pre-aligned molecules and determines the Tanimoto measures for the comparison. The similarity between the compounds and GTP- $\alpha-S$ was also accessed in terms of electrostatic potential with the program EON. Since the input molecules 
must already be aligned, the output result generated by ROCS program was used as input to EON program, then the molecules were ranked by the electrostatic Tanimoto (eT) score. The combination of ROCS and EON potentially yields compounds with similar shape and electrostatics. ${ }^{34,35}$

\section{Target based virtual screening methods}

FRED (Fast Exhaustive Docking, version 2.2.5), was used for docking purposes. The 3D structure of diguanylate cyclase PLED (PDB ID 2VON) used for docking was prepared with the MAKE RECEPTOR program. Water molecules and ligand were extracted from the protein structure, the hydrogens were added and a box of $20 \times 20 \times 20 \AA$ was generated and adjusted to encompass all residues within $5 \AA$ from the ligand coordinates, the GTP- $\alpha-S$ at the active site. Then the partial charges were generated. Default parameters were used for docking, with the following exceptions: inner and outer contour maps were disabled, thus the molecules were docked in the space between the box limits and the van der Waals radii active site atoms. Consensus scoring, with PLP, chemgauss 3 and chemscore scoring functions, was enabled during selection of the pose and, along with all remaining available functions (shapegauss, chemgauss2, chemscore, screenscore and zapbind), these were also used to score the selected pose. Predicted binding energy for each of these scoring functions was used in the analysis. H-bond acceptor constraints were imposed at the residues $\mathrm{N}^{335}$ and $\mathrm{D}^{344}$ in order to achieve an RMSD $<2 \AA$ in the re-docking experiments.

Consensus scores of ligand- and target-based virtual screening

The scaled-rank-by-number strategy was applied to rank and select candidate compounds for biochemical assays, where the final rank was generated by a simple sum of scaled score values of ROCS/EON and FRED for each compound.$^{36}$ Scaling of values was carried out according to the relation $\mathrm{X}_{\text {scaled }}=\left(\mathrm{x}-\mathrm{x}_{\min }\right) /\left(\mathrm{x}_{\max }-\mathrm{x}_{\text {min }}\right)$, where $\mathrm{X}_{\text {scaled }}$ is the scaled value, $x_{\max }$ and $x_{\min }$ are the maximum and minimum values outputted by a given program.

\section{Protein expression and purification}

The coding region of E. coli $\mathrm{YdeH}, P$. aeruginosa WspR and its isolated $\mathrm{GG}(\mathrm{D} / \mathrm{E}) \mathrm{EF}$ domain $\left(\mathrm{WspR}^{\mathrm{GG}(\mathrm{D} / \mathrm{E}) \mathrm{EF}}\right)$ were amplified by standard PCR using genomic DNA and cloned into the pET-SUMO expression plasmid, yielding $\mathrm{N}$-terminally $\mathrm{His}_{6}$-SUMO tag fusion proteins. Expression plasmids were transformed into E. coli BL21(DE3) and protein expression and purification followed similar protocols for all proteins. Transformed bacteria were grown in LB medium supplemented with kanamycin $50 \mathrm{mg} \mathrm{L}^{-1}$ at $37{ }^{\circ} \mathrm{C}$ to a cell density $0.6-0.8$ at $600 \mathrm{~nm}$. Protein expression was then induced with $0.1 \mathrm{mM}$ isopropyl $\beta$-D-1-thiogalactopyranoside (IPTG) for $16 \mathrm{~h}$ at $18{ }^{\circ} \mathrm{C}$. Cells were collected by centrifugation and resuspended in buffer $25 \mathrm{mM}$ Tris, $300 \mathrm{mM} \mathrm{NaCl}, 10 \mathrm{mM}$ imidazole, $\mathrm{pH}$ 7.5. Cells were disrupted by sonication and debris was removed by centrifugation at $40,000 \mathrm{~g}$ for $1 \mathrm{~h}$ at $4{ }^{\circ} \mathrm{C}$. Clear lysates were then loaded onto the Talon column (Talon ${ }^{\circledR}$ Superflow GE Life Sciences), pre-equilibrated with buffer $25 \mathrm{mM}$ Tris, $300 \mathrm{mM} \mathrm{NaCl}, 10 \mathrm{mM}$ imidazole, pH 7.5. The resin was washed exhaustively with this same buffer and the fusion protein was eluted with buffer $25 \mathrm{mM}$ Tris, $300 \mathrm{mM} \mathrm{NaCl}, 500 \mathrm{mM}$ imidazole, $\mathrm{pH}$ 7.5. The eluted fusion protein was treated with a recombinant $\mathrm{His}_{6}{ }_{-}$ULP1 protease (Ubl-specific protease 1) to remove the $\mathrm{N}$-terminal $\mathrm{His}_{6}$-SUMO tag. A second cobalt-affinity chromatography step was carried out to separate the cleaved tag-free protein from the $\mathrm{His}_{6}$-SUMO tag and $\mathrm{His}_{6}$-protease.

\section{DGC enzymatic assays}

The DGC activity was assayed using the PiPerTM pyrophosphate assay kit (Invitrogen). Pyrophosphate production was monitored by fluorescence of resorufin at $590 \mathrm{~nm}$. All kinetic measurements were carried out in buffer $100 \mathrm{mM}$ Tris $\mathrm{pH} 7.5,5 \mathrm{mM} \mathrm{MgCl}{ }_{2}$, at $25{ }^{\circ} \mathrm{C}$. For the calculation of $\mathrm{IC}_{50}(50 \%$ inhibitory concentration) values, $500 \mathrm{nM}$ of enzyme (WspR or YdeH) were assayed with $20 \mu \mathrm{M}$ GTP and varying inhibitor concentration (10 $\mu \mathrm{M}$ to $1 \mathrm{mM})$. Measurements were performed with a microplate reader SpectraMax (Molecular Devices). The percentage of inhibition was calculated according to the equation $\mathrm{I} \%=100 \times\left(1-\mathrm{v}_{\mathrm{i}} / \mathrm{v}_{0}\right)$, where $\mathrm{v}_{\mathrm{i}}$ and $\mathrm{v}_{0}$ are the initial velocities determined in the presence and absence of inhibitors, respectively. To calculate $\mathrm{IC}_{50}$ values, the dose-response curves were fitted to the equation $\mathrm{I} \%=\mathrm{A}_{0}+\left(\left(\mathrm{A}_{1}-\mathrm{A}_{0}\right) /\left(1+10^{\left(\log \mathrm{C}_{50}\right) \mathrm{h}}\right)\right)$, where $\mathrm{A}_{0}$ and $\mathrm{A}_{1}$ are the bottom and top plateaus of the curve, respectively. $\log \mathrm{IC}_{50}$ is the logarithm of the $\mathrm{IC}_{50}$ and $\mathrm{h}$ the Hill coefficient. All experiments were carried out at least in triplicates.

\section{Mass spectrometry experiments}

Electrospray ionization mass spectrometry (ESI-MS) was performed on a micrOTOF-II mass spectrometer (Bruker Daltonics), operated in positive ion mode. The capillary was set at $3500 \mathrm{~V}$ and high-voltage end-plate offset at $-500 \mathrm{~V}$. Broadband excitation was used to analyze 
a mass range from $m / 250-10000$ and with an acquisition time of $0.5 \mathrm{~min}^{\mathrm{sample}} \mathrm{e}^{-1}$. Samples were infused into the ESI source at $3 \mu \mathrm{L} \mathrm{min}{ }^{-1}$. Analysis of MS measurements was carried out using Compass 1.3 Data Analysis (Version 4.0, SP1) software (Bruker Daltonics). Agilent ESI tuning mix (Santa Clara) was used for an external enhanced quadratic mode calibration. The hexapole ion accumulation time was $3 \mathrm{~s}$. ESI-MS parameters were optimized using the isolated $\mathrm{WspR}^{\mathrm{GGDEF}}$ domain and GTP. Apparent $\mathrm{K}_{\mathrm{d}}$ measurements were carried based on direct detection and quantification of the gas phase ions of ligand bound and unbound protein. Assuming that the equilibrium ratio (R) between ligand-bound and -free protein concentrations measured by ESI-MS is equivalent to the equilibrium ratio in solution, dissociation constants $\left(\mathrm{K}_{\mathrm{d}}\right)$ were calculated via $\mathrm{K}_{\mathrm{d}}=\left([\mathrm{L}]_{0}-\frac{\mathrm{R}}{1+\mathrm{R}}[\mathrm{P}]_{0}\right) / \mathrm{R}$, where $[\mathrm{P}]_{0}$ and $[\mathrm{L}]_{0}$ are the initial protein and ligand concentrations, respectively.

Assays were performed with $500 \mathrm{nM} \mathrm{WspR}{ }^{\mathrm{GG}(\mathrm{D} / \mathrm{L}) \mathrm{EF}}$ and ligand concentration varying from 0.5 to $50 \mu \mathrm{M}$ (1 to 100 equivalent). To minimize buffer interference, protein (1 mM, in $25 \mathrm{mM}$ Tris, $300 \mathrm{mM} \mathrm{NaCl}$, pH 7.5 buffer) and ligand (100 mM, in 100\% DMSO) stocks were diluted 100 to 200 -fold in the assays. Samples were incubated at $25^{\circ} \mathrm{C}$ for $30 \mathrm{~min}$ prior to MS analysis. Competition experiments were carried out under the same conditions with equimolar concentrations of GTP and ligands $(10 \mu \mathrm{M})$.

\section{Ligand efficiency calculations}

Ligand efficiency (LE) is a useful parameter to guide the optimization of lead compounds. ${ }^{37}$ It measures the binding energy per atom of a ligand to a target protein via the relation $\mathrm{LE}=\Delta \mathrm{G} / \mathrm{n}$, where $\Delta \mathrm{G}=\mathrm{RT} \ln \mathrm{K}_{\mathrm{d}}$ is the free energy of ligand binding and $n$ is the number of non-hydrogen atoms. The $K_{d}$ values determined by mass spectrometry for each compound were used to calculate their LE towards WspR.

\section{Cell aggregation assay}

Transformed Escherichia coli bacteria with SUMO plasmids containing $\mathrm{YdeH}$ and WspR enzymes were grown under shaking in LB medium supplemented with kanamycin $50 \mathrm{mg} \mathrm{L}^{-1}$ at $37^{\circ} \mathrm{C}$ and $150 \mathrm{rpm}$. At a cell density corresponding to an absorbance of 0.8 at $600 \mathrm{~nm}$ IPTG $0.1 \mathrm{mM}$ was added to induce the DGC expression resulting in the biofilm formation, after $3 \mathrm{~h}$ a large number of aggregates were observed in the induced bacteria; the absence of IPTG resulted in no biofilm formation. The negative controls were established as bacteria transformed with empty SUMO vector, which does not form aggregates in both presence and absence of IPTG. To measure the ability of the drugs to prevent biofilm formation, drugs at $50 \mu \mathrm{M}$ were incubated for $30 \mathrm{~min}$ prior to IPTG addition. Optical phase contrast microscopy was used to observe the biofilm formation, $3 \mu \mathrm{L}$ of the media was placed in the slide and covered by the coverslip to flatten the sample. At least 20 different images were taken along a diagonal transect of the cover slips, with magnification of $200 \times$. Images were analyzed for biofilm area coverage using CellProfiler. ${ }^{38}$ The pipeline consisted of load images, enhance edges, apply threshold, identify primary objects, measure area occupied and export spreadsheet. The Student's $t$-test was applied with confidence level 0.05 and confidence interval of $99 \%$ to select the images. Experiments were carried out at least in triplicates.

\section{Results and Discussion}

\section{Virtual screening of diguanylate cyclase inhibitors}

Structural studies revealed that intercalated c-di-GMP dimers (c-di-GMP) $)_{2}$ bound to DGC I-sites prevent the productive encounter of $\mathrm{GG}(\mathrm{D} / \mathrm{E}) \mathrm{EF}$ active half-sites (A-site) by domain crosslinking (Figure 1A). ${ }^{16,40}$ Recognition of (c-di-GMP $)_{2}$ involves a widely conserved primary site (RxxD motif) at the GG(D/E)EF domain and secondary residues from other domains. The later are correctly oriented through inter- or intra-protein structural rearrangements upon c-di-GMP binding. For instance, two inactive forms of Caulobacter crescentus PleD show that (c-di-GMP) can either crosslink the $\mathrm{GG}(\mathrm{D} / \mathrm{E}) \mathrm{F}$ primary I-site with a CheY-like phosphoacceptor (REC domain) within the same protomer or with the neighboring $\mathrm{GG}(\mathrm{D} / \mathrm{E}) \mathrm{EF}$ domain of the dimeric enzyme. ${ }^{13,40}$ Given such plasticity and the complex nature of the ligand recognized by DGCs I-site, we target small molecules to the A-site of the enzyme. Residues involved in the recognition of GTP (Figure 1B) are highly conserved (Figure 1C), including $\mathrm{D}^{327}, \mathrm{~F}^{330}, \mathrm{~F}^{331}, \mathrm{~K}^{332}, \mathrm{~N}^{355}$, $\mathrm{D}^{344}, \mathrm{E}^{370}, \mathrm{~K}^{442}$ and $\mathrm{R}^{446}$ (C. crescentus PleD numbering).

The molecular docking strategy was defined based on the crystal structure of GTP- $\alpha$-S bound to the A-site of PleD (PDB ID 2V0N). The highly conserved residues $\mathrm{N}^{335}$ and $\mathrm{D}^{344}$ were set as constraints given their pivotal role in the recognition and specificity to GTP over other nucleotides by DGCs. ${ }^{29,41}$ Initial tests demonstrated that the FRED program was able to reproduce successfully the PleD-GTP- $\alpha-S$ complex with a docking energy of $-40 \mathrm{kcal} \mathrm{mol}^{-1}$, as calculated by the Chemgauss 3 scoring function. We then docked each of the 1,500 DrugBank compounds in 500 orientations at the PleD A-site (Figure 2A). From the total number of molecules in the database, $40 \%$ 

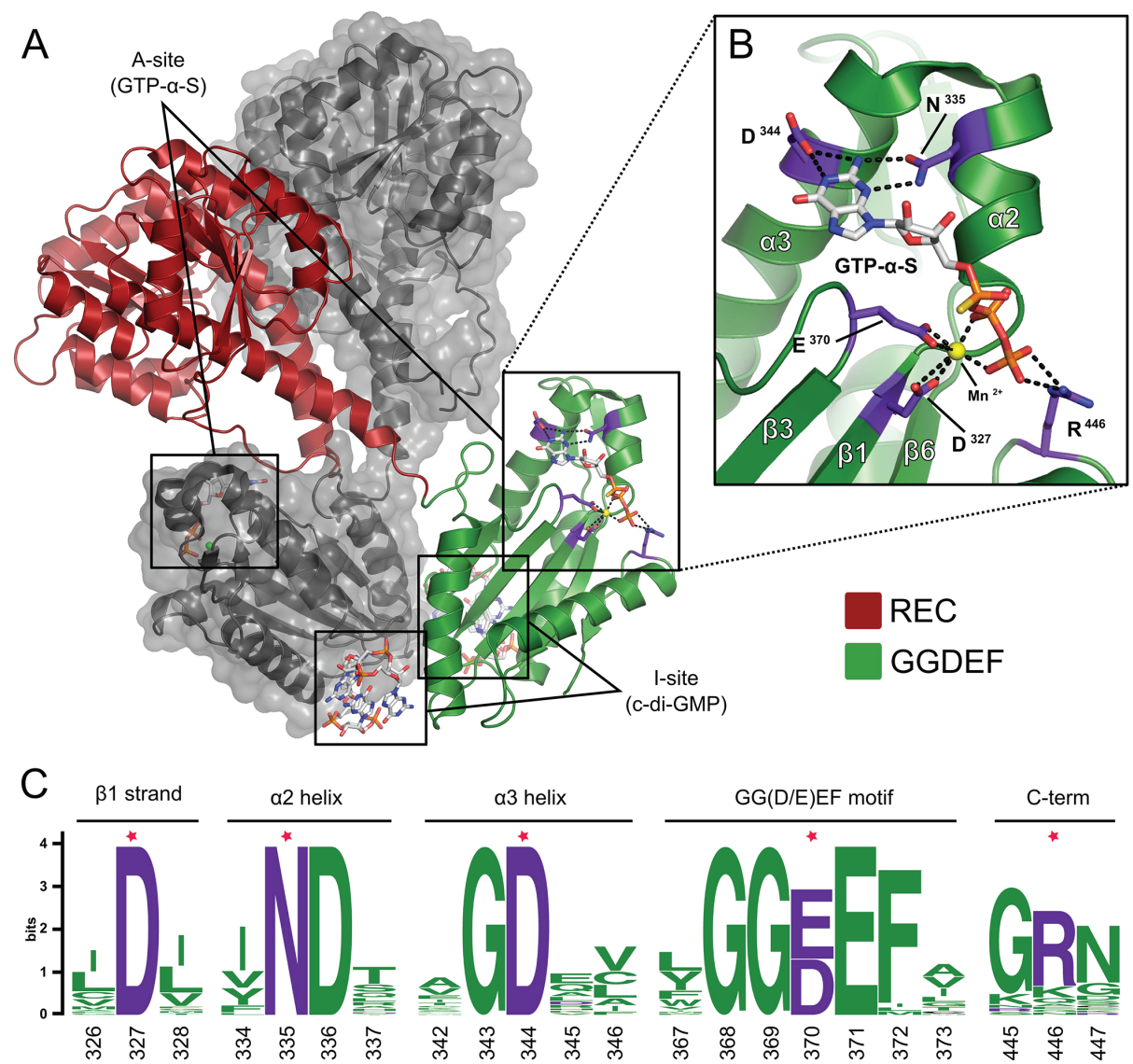

Figure 1. Structure of the DGC PleD complexed with GTP- $\alpha$-S used for docking. (A) Cartoon representation of the dimeric PleD structure (PDB ID 2V0N). One of the protomers is colored gray, and a semi-transparent water-accessible surface is shown. The N-terminal REC and GG(D/E)EF domains of the other protomer are colored red and green, respectively. Residues interacting with GTP- $\alpha-\mathrm{S}$ at the A-site of the GG(D/E)EF domain are colored purple. A-site and I-site of both protomers are highlighted in black boxes. (B) Details of the A-site of PleD bound to GTP- $\alpha$-S. Black dashed lines indicate interactions between PleD residues and GTP- $\alpha-S$. The solid yellow sphere represents a $\mathrm{Mn}^{2+}$ ion interacting with PleD E ${ }^{370}$ and $\mathrm{D}^{327}$ side chains and GTP- $\alpha-S$ phosphate groups. Colors are consistent with panel (A). (C) Logo representation of a multiple sequence alignment of DGC homologues covering all motifs involved in the recognition of GTP- $\alpha$-S. Sequences aligned were selected from the PFAM database and the logo were generated with Skylign. ${ }^{39}$

were successfully docked, and $5.9 \%$ showed calculated binding energy higher than re-docking of GTP- $\alpha$-S. Alternatively, we searched compounds similar to GTP in the DrugBank database. Indeed, ligand-based methods are valuable tools in virtual screening campaigns, and usually present comparable performances to other computational methods. ${ }^{42,43}$ Distribution of shape and electrostatics similarity using ST (shape Tanimoto) and eT (electrostatic Tanimoto) scores, as respectively implemented in ROCS and EON programs, are shown in Figures $2 \mathrm{~B}$ and $2 \mathrm{C}$.

The final rank of molecules was obtained by combining the individual target- and ligand-based virtual screening results in a consensus scoring function named scaledrank-by-number (Figure 2D). This strategy is promising for finding inhibitors with innovative scaffolds while conserving similar properties to the natural DGCs substrate. The 200 highest-score compounds were visually inspected and 10 were selected for biochemical assays following the criteria of GG(D/E)EF A-site occupancy, hydrogen bonding network, constraint matching and molecular diversity (Figure 3).

Functional tests on the selected compounds from the virtual screening

The selected compounds were tested against the diguanylate cyclases WspR and YdeH from P. aeruginosa and $E$. coli, respectively. Such approach is advantageous with respect to the identification of inhibitors targeting conserved binding motifs rather than species-specific binding sites, possibly leading to anti-biofilm compounds with broad-spectrum. Since c-di-GMP usually co-purifies with $\mathrm{WspR},{ }^{16}$ we generated an I-site mutated version $\left(\mathrm{R}^{242} \mathrm{~A}\right)$ of the enzyme to simplify the biochemical analyzes. Different from WspR, YdeH exhibits weak product inhibition, and recent results indicate that its divergent I-site could be involved in c-di-GMP-mediated protein interaction, rather than enzyme allosteric regulation. ${ }^{41} \mathrm{In}$ 
A
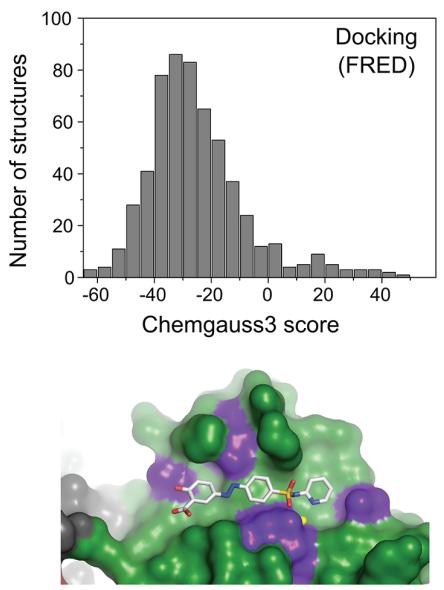

D

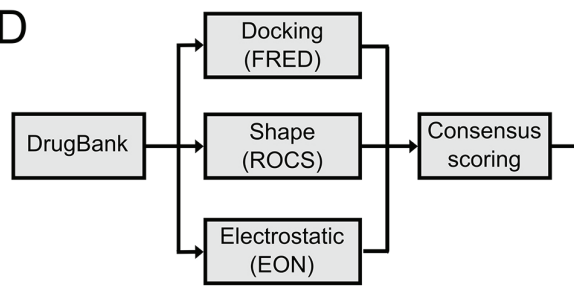

C
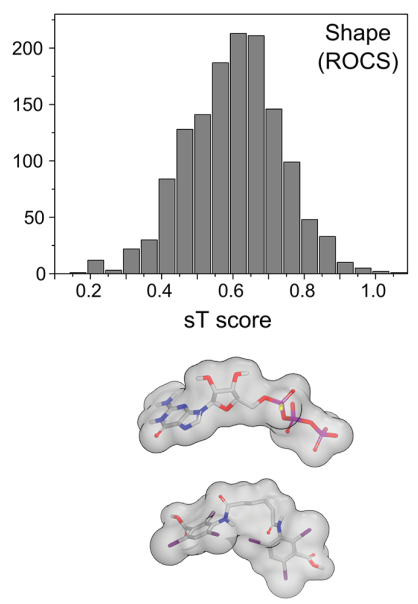
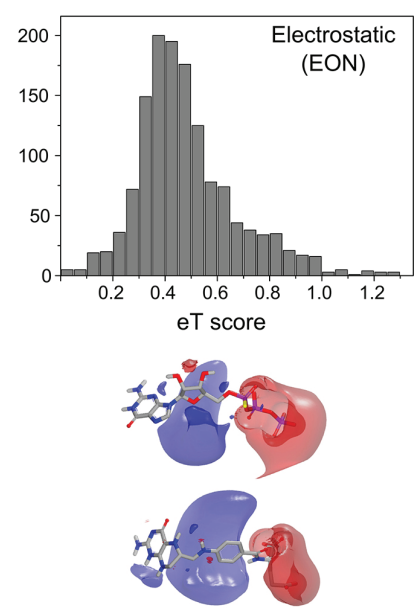

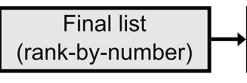

Top 100 (visual inspection) Compounds to
be assayed

Figure 2. Virtual screening of potential DGC inhibitors. Histograms of binding energy, calculated with FRED (A), shape similarity calculated by ROCS (B) and electrostatic similarity calculated by the EON (C). (D) Flowchart of the virtual screening strategy employed for selecting compounds.<smiles>COC1[C@@H](OC(N)=O)[C@@H](O)C(Oc2ccc3c(O)c(NC(=O)c4ccc(O)c(CC=C(C)C)c4)c(=O)oc3c2C)OC1(C)C</smiles><smiles>C=CC1=C(C(=O)O)N2C(=O)[C@@H](NC(=O)/C(=N\OCC(=O)O)c3csc(N)n3)C2SC1</smiles><smiles>O=C(CCCCC(=O)Nc1c(I)cc(I)c(C(=O)O)c1I)Nc1c(I)cc(I)cc1I</smiles>
lodipamine<smiles>Nc1nc(=O)c2ncn(COCCO)c2[nH]1</smiles><smiles>C=C(CN=CC(=O)OCc1ccccc1)Nc1ccccc1</smiles><smiles>CC(=O)NC(CCC(=O)O)C(=O)O</smiles><smiles>CC=C(C)N=Nc1ccc(S(=O)(=O)Nc2ccccn2)cc1</smiles>
Acyclovir<smiles>Nc1ccc(S(=O)(=O)Nc2ncccn2)cc1</smiles>

Sulfadiazine

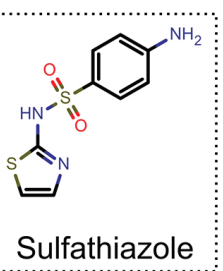

Figure 3. Molecular structures of the compounds selected for biochemical and functional assays. Sulfathiazole, an anti-biofilm agent previously identified, ${ }^{26}$ is highlighted in a dashed black box.

this way, we ensured the enzymes used in our assays were not co-purified with c-di-GMP or susceptible to product inhibition along the in vitro enzymatic reactions.

In initial enzymatic assays carried out with the PiPer ${ }^{\mathrm{TM}}$ pyrophosphate kit, cefixime, biotin, sulfathiazole and acyclovir at a concentration of $1 \mathrm{mM}$ did not inhibit either YdeH or WspR. While iodipamide and sulfadiazine displayed partial inhibition, sulfasalazine, folic acid, novobiocin and eprosartan completely inhibited the DGC activity of both enzymes. $\mathrm{IC}_{50}$ values fluctuated in the mid-high micromolar range (Table 1 and Figure 4).
Due to the strategy employed in the virtual screening, we expected to observe similar activities of the selected compounds against both enzymes. Accordingly, novobiocin, sulfasalazine, and folic acid presented an increasing potency trend for both $\mathrm{YdeH}$ and $\mathrm{WspR}$, with $\mathrm{IC}_{50}$ values slightly lower in the case of $\mathrm{YdeH}$. The only exception is eprosartan, which turned to be the most potent inhibitor identified for $\mathrm{WspR}\left(\mathrm{IC}_{50}\right.$ of $\left.170 \pm 15 \mu \mathrm{M}\right)$ while it weakly inhibited YdeH $\left(\mathrm{IC}_{50}\right.$ of $\left.888 \pm 70 \mu \mathrm{M}\right)$. Although key residues involved in GTP recognition are strictly conserved in both WspR and YdeH active sites, different A-site peripheral residues, such 
Table 1. $\mathrm{IC}_{50}$ for $\mathrm{YdeH}$ and $\mathrm{WspR}, \mathrm{K}_{\mathrm{d}}^{\text {app }}$ values for the isolated $\mathrm{GG}(\mathrm{D} / \mathrm{E}) \mathrm{F}$ domain of WspR (WspR ${ }^{\mathrm{GG}(\mathrm{D} / \mathrm{F}) \mathrm{EF}}$ ) domain and calculated ligand efficiency (LE)

\begin{tabular}{|c|c|c|c|c|}
\hline Compound & $\mathrm{YdeH} \mathrm{IC}_{50} / \mu \mathrm{M}$ & $\mathrm{WspR} \mathrm{IC}_{50} / \mu \mathrm{M}$ & $\mathrm{WspR}^{\mathrm{GG(D/F)EF}} \mathrm{K}_{\mathrm{d}} / \mu \mathrm{M}$ & $\begin{array}{l}\text { WspR }{ }^{\mathrm{GG}(\mathrm{D} / \mathrm{E}) \mathrm{EF}} \mathrm{LE} / \\
\left(\mathrm{kcal} \mathrm{mol}^{-1} \text { atom }^{-1}\right)\end{array}$ \\
\hline Sulfasalazine & $200 \pm 20$ & $360 \pm 50$ & $40 \pm 10$ & 0.21 \\
\hline Folic acid & $430 \pm 20$ & $500 \pm 70$ & $50 \pm 10$ & 0.18 \\
\hline Eprosartan & $890 \pm 70$ & $170 \pm 20$ & $40 \pm 10$ & 0.21 \\
\hline Novobiocin & $140 \pm 20$ & $220 \pm 30$ & $130 \pm 30$ & 0.13 \\
\hline Iodipamide & ND & ND & $130 \pm 30$ & 0.15 \\
\hline Sulfadiazine & ND & ND & $310 \pm 40$ & 0.28 \\
\hline Sulfathiazole & ND & ND & $490 \pm 80$ & 0.26 \\
\hline GTP $^{a}$ & & & $7 \pm 4$ & 0.22 \\
\hline
\end{tabular}

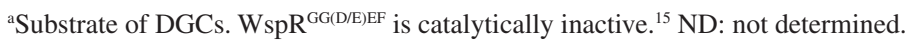
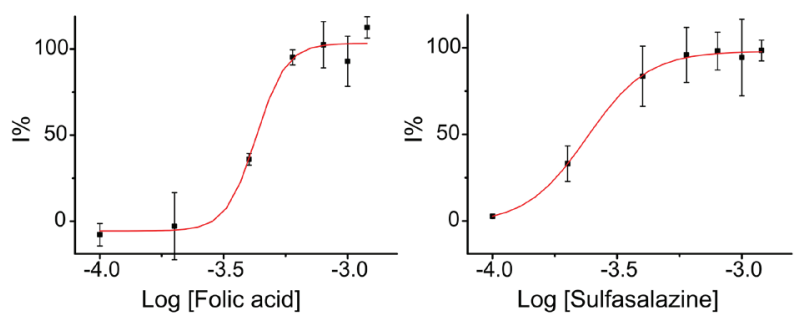

Figure 4. Representative dose-response curves of the compounds tested. Inhibition of WspR activity by sulfasalazine (right) and YdeH activity of folic acid (left) are shown. Kinetic experiments were carried out with a DGC concentration of $500 \mathrm{nM}$.

as WspR $\mathrm{Y}^{212}$ and $\mathrm{S}^{215}$ (YdeH R ${ }^{168}$ and $\mathrm{L}^{171}$, respectively), might account for this differential activity.

We then turn to ESI-MS to measure apparent dissociation constants $\left(\mathrm{K}_{\mathrm{d}}\right)$ and confirm that inhibition was achieved by the interaction of compounds with the DGC, not protein adsorption to drug aggregates. ESI-MS is particularly useful for discovery and characterization of lead compounds since it is highly sensitive and directly probe weak non-covalent protein-ligand interactions ${ }^{44}$ By using a highly stable isolated GG(D/E)EF domain construct of WspR (WspR $\left.{ }^{\mathrm{GG}(\mathrm{D} / \mathrm{F}) \mathrm{EF}}\right),{ }^{15}$ the $\mathrm{K}_{\mathrm{d}}$ measured for sulfasalazine, folic acid, eprosartan, novobiocin, iodipamide, sulfadiazine and sulfathiazole ranged from 37 to $493 \mu \mathrm{M}$ (Table 1 and Figure 5). Remarkably, GTP displayed a $\mathrm{K}_{d}$ compatible with previously reported WspR Michaelis-Menten constant, ${ }^{15}$ $7.3 \mu \mathrm{M}$ (ESI-MS) and 5.8 $\mu \mathrm{M}$ (enzyme assay), respectively, demonstrating the robustness of the method and reliability of the results obtained. The high sensitivity of ESI-MS enabled us to measure interaction constants for iodipamine, sulfadiazine and sulfathiazole, compounds that poorly inhibited the DGCs.

To further probe the binding site of the identified inhibitors, competition experiments were carried out with GTP. If a compound binds to a different location than the GG(D/E)EF A-site, peaks compatible with the mass of ternary WspR $\mathrm{R}^{\mathrm{GG}(\mathrm{D} / \mathrm{E}) \mathrm{EF}}$-ligand-GTP complexes would appear on ESI-MS experiments. Mass spectra of $\mathrm{WspR}^{\mathrm{GG}(\mathrm{D} / \mathrm{E}) \mathrm{EF}}$ $(500 \mathrm{nM})$ pre-incubated with equimolar concentrations of GTP and inhibitors $(10 \mu \mathrm{M})$ presented only peaks related to $\mathrm{WspR}^{\mathrm{GG}(\mathrm{D} / \mathrm{E}) \mathrm{EF}}-\mathrm{GTP}$ complex, demonstrating GTP displaces all the inhibitors out of $\mathrm{GG}(\mathrm{D} / \mathrm{E}) \mathrm{EF}$ domain A-site (Figure 5J).

Although the biochemical assays we performed are limited to compounds covering a reduced number of functional groups, we observed an interesting structureactivity relationship. For instance, the presence of a common benzenesulfonamide moiety in the compounds with the highest ligand efficiency (Table 1), sulfadiazine, sulfathiazole and sulfasalazine, indicates that this moiety may be engaged in specific interactions, and thus represent the most promising compounds for potency optimization in this series. ${ }^{37,45}$ Docking results predict that the benzenesulfonamide moiety occupy the triphosphate-binding pocket of DGCs A-site, where protein-ligand interactions are potentially mediated by the magnesium ion (Figure 6). Such metal coordination behavior was previously reported for several sulfonamide derivatives ${ }^{46}$ The importance of the triphosphate-binding pocket for molecular recognition is further supported by the inefficacy of the guanine-derivative drug acyclovir as a DGC inhibitor and its known mechanism of action. To potentially inhibit viral DNA polymerases, acyclovir undergoes a selective incorporation of triphosphate by viral and cellular kinases. ${ }^{47}$ On the other hand, the predict interaction of the 5-aminosalicylic acid group of sulfasalazine with the conserved $\mathrm{D}^{344}$ amino acid at the guanine binding site (Figure 6) is probably responsible for the 8-fold decrease in $\mathrm{K}_{\mathrm{d}}$ compared to sulfadiazine, indicating that exploration of both triphosphate and guanine sites are essential for the development of high affinity DGC inhibitors. 

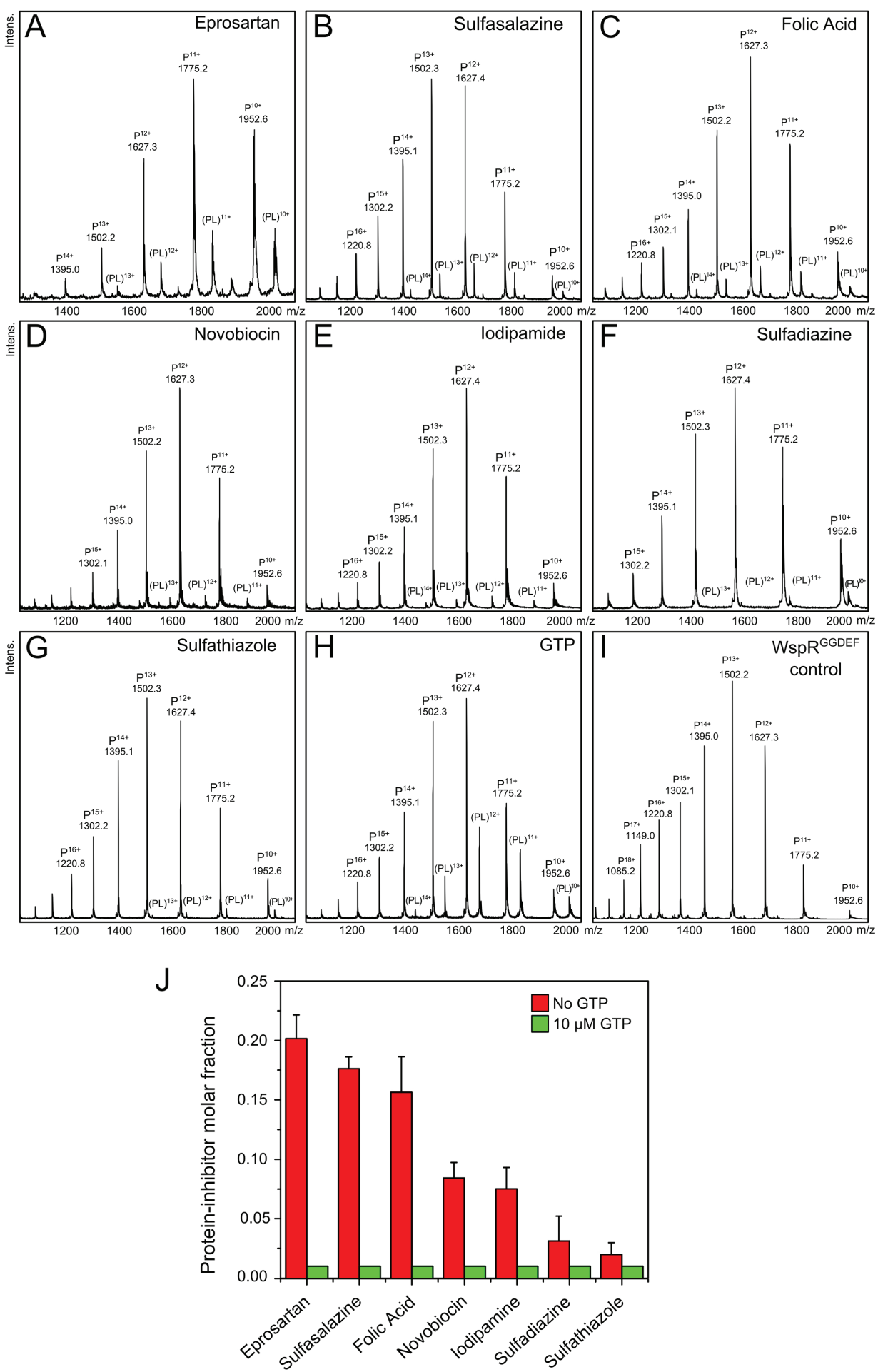

Figure 5. Detection of protein-compound interaction by ESI-MS. (A-H) ESI mass spectrum obtained in positive ion mode for an aqueous solution containing $500 \mathrm{nM} \mathrm{WspR}^{\mathrm{GG}(\mathrm{D} / \mathrm{E}) \mathrm{EF}}$ and $10 \mu \mathrm{M}$ of compounds. PL indicates peaks corresponding to protein-ligand complexes; (I) control ESI mass spectrum of WspR ${ }^{\mathrm{GG}(\mathrm{D} / \mathrm{E}) \mathrm{EF} \text {; }}$ (J) molar fraction of the protein-compound complexes in the presence (gray) or absence (black) of equimolar concentrations of the competitor GTP. Experiments were carried out at least in triplicates.

Bacterial cells usually communicate through direct contact and many species display a biofilm type of organization, even when grown in liquid media. ${ }^{48} E$. coli biofilm formation was stimulated by inducing expression of cells (BL21-DE3 strain) harboring plasmids with the DGCs WspR or YdeH under the control of T7lac promoter.

The aggregation of bacteria in solution was explored by Massie et al. ${ }^{49}$ to analyze the biofilm formation under 

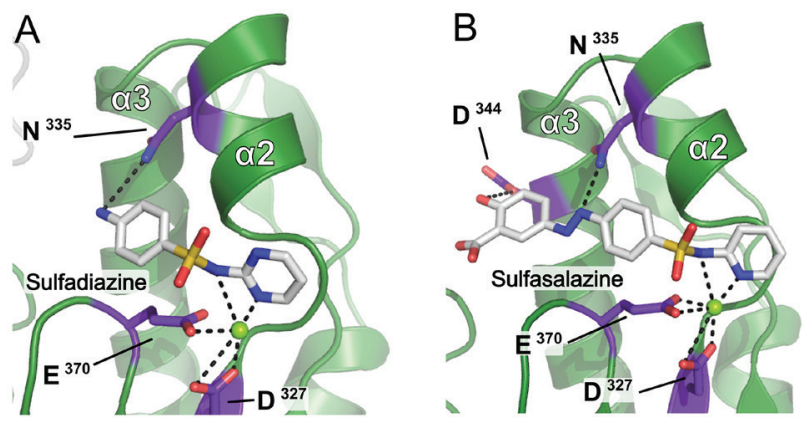

Figure 6. Docking poses of sulfadiazine (A) and sulfasalazine (B) at the A-Site of PleD. Pyridine nitrogen coordinates the $\mathrm{Mg}^{2+}$ ion (green sphere). PleD cartoon representation and side chain residues are colored as in Figure 1. Black dashed lines indicate interactions between PleD residues, $\mathrm{Mg}^{2+}$ ion and docked ligands.

shaking condition; in another study, Vibrio cholerae bacteria was used to overexpressed different DGCs enzymes. In this study, the DGCs WspR and YdeH were overexpressed in E. coli with different IPTG concentrations and aggregation formation was observed by optical phasecontrast microscopy. Empty SUMO vector was used as negative control (Figures 7G, 7H and 7I), even upon addition of IPTG resulted only single cells. On the other hand, aggregates were clearly visible upon addition of $0.1 \mathrm{mM}$ IPTG (Figures 7A and 7D) with bacterial harboring DGCs vectors containing WspR and YdeH enzymes, established as positive controls, which production was probably triggered by elevated c-di-GMP levels. Since phase-contrast microscopy enables direct visualization of cell aggregates, we implement a pipeline for detection and measurement of relative aggregate-covered area (RACA) using CellProfiler software. ${ }^{38}$ RACA is a good biofilm quantification index since it enables the evaluation of cell aggregate population and size distributions simultaneously. IPTG concentration of $0.1 \mathrm{mM}$ consistently yielded cell aggregates ranging from 5 to $50 \mu \mathrm{m}$. RACA correlate well with increasing of IPTG concentrations.

The DGC inhibitors identified by biochemical assays were tested to check their ability to interfere in biofilm formation by phase-contrast microscopy RACA analysis. In these assays, we used fixed concentrations of IPTG $(0.1 \mathrm{mM})$ and inhibitors $(50 \mu \mathrm{M})$. Since folic acid is unable to cross the bacterial cell wall by diffusion or active transport,${ }^{50}$ we exclude this compound from further analysis. Compounds tested at $50 \mu \mathrm{M}$ did not interfere with bacterial growth (data not shown); the only exception was novobiocin, an antibiotic that targets bacterial DNA gyrase..$^{51}$ On the other hand, a marked decrease in bacterial aggregation, quantified by RACA, was observed (Figure 7J).

Overall, inhibitors were more potent in bacteria transformed with YdeH plasmid, where a concentration of $50 \mu \mathrm{M}$ was able to prevent cell aggregation by 68 to $99 \%$ (eprosartan and sulfathiazole, respectively) (Figures 7B, 7C, 7E and 7F). The drug that inhibited efficiently cell aggregation induced by WspR was eprosartan, with a reduction of approximately $90 \%$. These results correlate very well with the inhibitory activity and affinity of the compounds against WspR and YdeH measured in the biochemical assays, suggesting that a reduction in intracellular c-di-GMP levels promoted either by compounds that direct inhibits DGCs or by interference with the bacterial nucleotide pool, might be responsible for the reducing of cell aggregates. Finally, in agreement with previous results reported by Antoniani et al. ${ }^{26,27}$ the
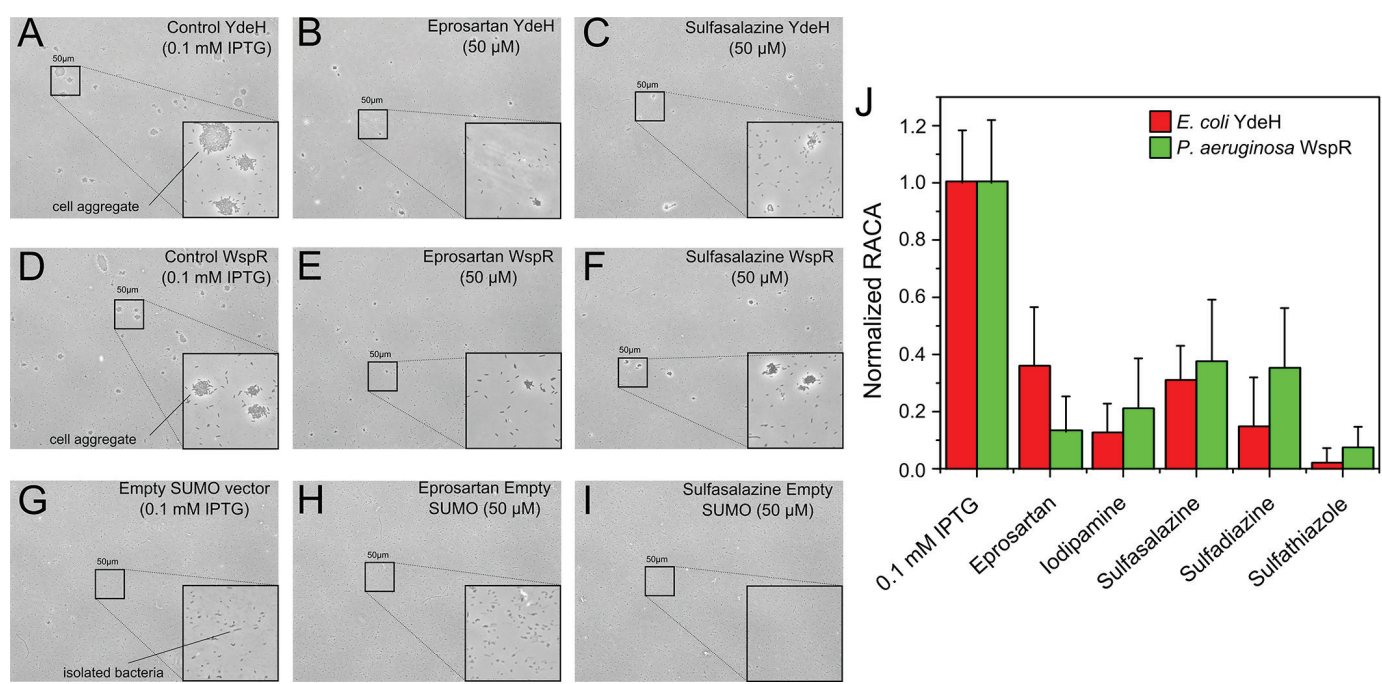

Figure 7. The ability of the DGC inhibitors to prevent biofilm formation. The addition of IPTG $0.1 \mathrm{mM}$ induces biofilm formation A and D (positive control), transformed bacteria with empty vector does not form biofilm (negative control). DGC inhibitors at $50 \mu \mathrm{M}$ were incubated for 30 min prior to IPTG addition. Eprosartan (B, E and H), and sulfasalazine are shown as examples (C, F and I). Relative aggregate-covered area (RACA) was measured for evaluating the biofilm reduction in E. coli transformed with plasmid containing YdeH and WspR genes (J). Experiments were carried out at least in triplicates. 
potent anti-biofilm agent sulfathiazole almost completely abolished DGC-induced cell aggregation, validating our assays.

Growing evidence has established that high c-di-GMP intracellular concentrations inhibit bacterial motility while stimulates the production of extracellular polysaccharides and other adhesion factors, leading to a transition between planktonic and biofilm lifestyles. ${ }^{12}$ Given that biofilm formation appears to be an important virulence factor underlying several chronic bacterial infections, ${ }^{52}$ targeting c-di-GMP mediated signaling might represent an attractive anti-microbial therapy. In this study, we discovered antiinflammatory and anti-hypertensive compounds selected from the FDA-approved drug database that displayed in vitro inhibitory activity in the micromolar range against DGCs enzymes from E. coli (YdeH) and P. aeruginosa (WspR).

The combined strategy of computational and experimental approaches represents an effective tool for rational drug design, usually yielding results comparable to high throughput screening (HTS) campaigns. Sambanthamoorthy et al. ${ }^{24}$ used $V$. cholera carrying a luciferase c-di-GMP-responsive reporter plasmid to screen potential anti-biofilm agents and identified seven DGCs inhibitor out of 66,000 tested. Here, out of the ten compounds selected from the DrugBank database through a hybrid target- and ligand-based virtual screen consensual strategy, eprosartan and sulfasalazine presented similar DGCs inhibition to the compounds selected by HTS. In a recent study, potent DGCs inhibitors were identified through a 3D-pharmacophore model-based virtual screening campaign, ${ }^{53}$ further supporting the effectiveness of computational methods for the identification of c-di-GMP biosynthesis antagonists.

Amongst the molecules we selected for functional and biochemical evaluations, there are three sulfonamidecontaining drugs, sulfadiazine, sulfasalazine and the previously described anti-biofilm agent sulfathiazole. In accordance to the results observed for sulfathiazole, the structurally-related molecule sulfadiazine (Figure 3 ) failed to inhibit the DGC activity of purified P. aeruginosa WspR and E. coli $\mathrm{YdeH}$ in biochemical assays. Antoniani et al. ${ }^{26}$ proposed that sulfathiazole inhibit c-di-GMP biosynthesis in vivo and, consequently, biofilm formation due to its antimetabolite activity. In fact, both sulfonamide antibiotics, as well as sulfasalazine, are inhibitors of the tetrahydrofolate biosynthesis via interaction with the dihydropteroate synthase, interfering with intracellular GTP pools. ${ }^{54}$ Surprisingly, we detected a weak binding of sulfathiazole and sulfadiazine to the isolated $\mathrm{WspR}^{\mathrm{GG}(\mathrm{D} / \mathrm{F}) \mathrm{EF}}$ domain using high sensitivity mass spectrometry. Although it is unlikely that the anti-biofilm effects of sulfathiazole, and potentially sulfadiazine, involve direct inhibition of DGCs, their predict mode of interaction with the A-site of GG(D/E)EF domains (Figure 6), combined with a high ligand efficiency (Table 1), suggest that the sulfonamide moiety represents a useful structure for ligand potency optimization. In fact, the presence of a 5-aminosalicylic acid group in sulfasalazine, probably occupying the DGC guanine binding site, enhanced 8-fold the ligand affinity towards $\mathrm{WspR}^{\mathrm{GG}(\mathrm{D} / \mathrm{E}) \mathrm{EF}}$ and allowed the direct detection of DGC catalytic activity inhibition $\left(\mathrm{IC}_{50}\right.$ of $358 \mu \mathrm{M}$ for P. aeruginosa $\mathrm{WspR}$ ). Notably, sulfasalazine shares a remarkable structural similarity with other DGCs inhibitors with potent anti-biofilm properties (Figure 8). ${ }^{24}$
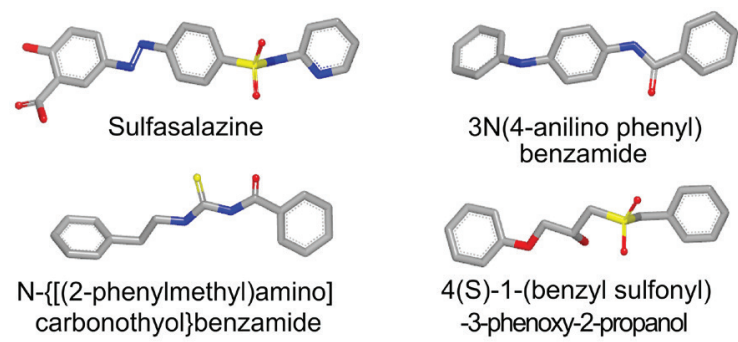

Figure 8. Molecular structures in similar conformations of sulfasalazine and three DGC inhibitors identified by HTS. ${ }^{24}$

We also selected an anti-hypertensive drug (eprosartan) from our virtual screening, which revealed itself as an inhibitor of bacterial DGCs. Eprosartan and other angiotensin receptor blockers (ARBs) exert their pharmacological properties acting as a specific antagonist of type 1 angiotensin II receptor (AT1R), one of the core components of the renin-angiotensin system (RAS). ${ }^{55}$ Classically, chronic RAS activation through AT1R leads to increased blood pressure, renal salt retention, aldosterone release and sympathetic nervous system activation. ${ }^{56}$ Locally, stimulation of RASs may influence diverse physiological outputs, especially inflammatory processes through AT1R-mediated stimulation of neutrophil migration ${ }^{57}$ and activation of the pro-inflammatory effector NF- $\mathrm{KB}$ (nuclear factor- $\mathrm{\kappa B}$ ) in phagocytes,$^{58}$ amongst others. AT1R blockers were effective in counteracting the deleterious inflammatory responses elicited by some bacterial infections, including acute respiratory distress syndrome (ARDS) triggered by Bordetella bronchiseptica ${ }^{59}$ and lung colonization by $P$. aeruginosa.$^{60}$ Experiments with rat models of lung injury caused by instillation of $P$. aeruginosa or the induction of ARDS in Wistar rats challenged by Bordetella bronchiseptica showed that administration of losartan (same molecular class of eprosartan) significantly inhibited the recruitment of neutrophil to lung. ${ }^{60}$ Captopril and saralasin, drugs that interfere with RASs activation through distinct modes of action, were unable to elicit the 
same response, suggesting that losartan effect is unrelated to its ability to antagonize ATR1. The structure-related ARBs ibersartan, candesartan, and valsartan presented similar anti-inflammatory response, ${ }^{60}$ further supporting a specific effect of this drug family. Surprisingly, despite reducing the recruitment of neutrophils and lacking bacteriostatic or bactericidal properties, losartan augmented bacterial clearance. ${ }^{60}$ Once c-di-GMP is strong effector of the immune response ${ }^{61}$ and interference with c-di-GMP signaling promotes bacterial clearance, ${ }^{23}$ our findings about eprosartan suggest that the effects of ARBs on these infections could be related to direct or indirect inhibition of c-di-GMP biosynthesis. Further investigations are required to evaluate this hypothesis.

Recent studies using cell-based high throughput screening identified molecules that impair c-di-GMP intracellular levels through direct inhibition of $\mathrm{DGCs}^{24}$ or by interference with nucleotide substrate pools. ${ }^{26,27}$ In this report, we used an in silico strategy to identify DGC inhibitors. Since the selected compounds are FDA-approved drugs, their scaffolds lie within a privileged chemical-biology space, where their pharmacokinetic properties and toxicological profiles are well established. The possibility of repositioning existing drugs for new indications is particularly attractive since it potentially reduces the expensive costs associated with hit compounds in early-stage tests. ${ }^{62}$ In this way, optimization of the compounds identified in this study could yield potent DGC inhibitors harboring drug-like properties with the potential to counteract biofilm-based chronic infections.

\section{Conclusions}

The progress in the development of anti-biofilms agent could be the drug-reposition strategy from the FDA-approved drugs, computational methods of targetand ligand-based virtual screening allow successful identification of DGCs inhibitors displaying potency values in the micromolar range, $\mathrm{IC}_{50}$ from 140 to $890 \mu \mathrm{M}$, determined by enzyme inhibition biochemical assays. The inhibitors target the GTP active site as confirmed by competitive assays using mass spectrometry, which was used also to measure affinity, $K_{d}$ values from 40 to $490 \mu \mathrm{M}$. The benzenesulfonamide moiety present in the drugs sulfathiazole, sulfadiazine and sulfasalazine were identified as a potential scaffold useful for inhibitor potency optimization. The most promising compounds, reported for the first time, are the anti-inflammatory sulfasalazine and anti-hypertensive eprosartan, that showed anti-biofilm activity and also inhibits the enzymes responsible for c-di-GMP biosynthesis, the signaling molecule for bacterial biofilm formation. Therefore, it may be useful for the development of adjutants for antibacterial infection treatments.

\section{Acknowledgments}

We are grateful to the OpenEye Scientific Software for providing us with their program package. Our study was supported by Fundação de Amparo à Pesquisa do Estado de São Paulo under Grant 2009/13238-0 (to M. V. A. S. N.) and Fundação de Amparo à Pesquisa do Estado de São Paulo Fellowship 2010/19109-4 (to H. J. W.).

\section{References}

1. Hall-Stoodley, L.; Costerton, J. W.; Stoodley, P.; Nat. Rev. Microbiol. 2004, 2, 95.

2. Anderl, J. N.; Franklin, M. J.; Stewart, P. S.; Antimicrob. Agents Chemother. 2000, 44, 1818.

3. Meredith, H. R.; Srimani, J. K.; Lee, A. J.; Lopatkin, A. J.; You, L.; Nat. Chem. Biol. 2015, 11, 182.

4. Jamal, M.; Tasneem, U.; Hussain, T.; Andleeb, S.; Res. \& Rev.: J. Microbiol. Biotechnol. 2015, 4, 1.

5. Parsek, M. R.; Singh, P. K.; Annu. Rev. Microbiol. 2003, 57, 677.

6. Tamayo, R.; Pratt, J. T.; Camilli, A.; Annu. Rev. Microbiol. 2007, 61,131 .

7. Wilkins, M.; Hall-Stoodley, L.; Allan, R. N.; Faust, S. N.; J. Infect. 2014, 69 Suppl 1, S47.

8. Christensen, L. D.; van Gennip, M.; Jakobsen, T. H.; Alhede, M.; Hougen, H. P.; Høiby, N.; Bjarnsholt, T.; Givskov, M.; J. Antimicrob. Chemother. 2012, 67, 1198.

9. Caly, D. L.; Bellini, D.; Walsh, M. A.; Maxwell Dow, J.; Ryan, R. P.; Curr. Pharm. Des. 2015, 21, 12.

10. Römling, U.; Balsalobre, C.; J. Intern. Med. 2012, 272, 541.

11. Hengge, R.; Nat. Rev. Microbiol. 2009, 7, 263.

12. Römling, U.; Galperin, M. Y.; Gomelsky, M.; Microbiol. Mol. Biol. Rev. 2013, 77, 1.

13. Wassmann, P.; Chan, C.; Paul, R.; Beck, A.; Heerklotz, H.; Jenal, U.; Schirmer, T.; Structure 2007, 15, 915.

14. Chan, C.; Paul, R.; Samoray, D.; Amiot, N. C.; Giese, B.; Jenal, U.; Schirmer, T.; Proc. Natl. Acad. Sci. U. S. A. 2004, 101, 17084.

15. De, N.; Navarro, M. V. A. S.; Raghavan, R. V.; Sondermann, H.; J. Mol. Biol. 2009, 393, 619.

16. De, N.; Pirruccello, M.; Krasteva, P. V.; Bae, N.; Raghavan, R. V.; Sondermann, H.; PLoS Biol. 2008, 6, e67.

17. Kulasakara, H.; Lee, V.; Brencic, A.; Liberati, N.; Urbach, J.; Miyata, S.; Lee, D. G.; Neely, A. N.; Hyodo, M.; Hayakawa, Y.; Ausubel, F. M.; Lory, S.; Proc. Natl. Acad. Sci. U. S. A. 2006, 103, 2839. 
18. Beyhan, S.; Tischler, A. D.; Camilli, A.; Yildiz, F. H.; J. Bacteriol. 2006, 188, 3600.

19. Ahmad, I.; Lamprokostopoulou, A.; Le Guyon, S.; Streck, E.; Barthel, M.; Peters, V.; Hardt, W.-D.; Römling, U.; PLoS One 2011, 6, e28351.

20. Barraud, N.; Schleheck, D.; Klebensberger, J.; Webb, J. S.; Hassett, D. J.; Rice, S. A.; Kjelleberg, S.; J. Bacteriol. 2009, $191,7333$.

21. Sauer, K.; Cullen, M. C.; Rickard, A. H.; Zeef, L. A. H.; Davies, D. G.; Gilbert, P.; J. Bacteriol. 2004, 186, 7312.

22. Gjermansen, M.; Ragas, P.; Sternberg, C.; Molin, S.; TolkerNielsen, T.; Environ. Microbiol. 2005, 7, 894.

23. Christensen, L. D.; van Gennip, M.; Rybtke, M. T.; Wu, H.; Chiang, W.-C.; Alhede, M.; Høiby, N.; Nielsen, T. E.; Givskov, M.; Tolker-Nielsen, T.; Infect. Immun. 2013, 81, 2705.

24. Sambanthamoorthy, K.; Sloup, R. E.; Parashar, V.; Smith, J. M.; Kim, E. E.; Semmelhack, M. F.; Neiditch, M. B.; Waters, C. M.; Antimicrob. Agents Chemother. 2012, 56, 5202.

25. Sambanthamoorthy, K.; Luo, C.; Pattabiraman, N.; Feng, X.; Koestler, B.; Waters, C. M.; Palys, T. J.; Biofouling 2014, 30, 17.

26. Antoniani, D.; Bocci, P.; Maciag, A.; Raffaelli, N.; Landini, P.; Appl. Microbiol. Biotechnol. 2010, 85, 1095.

27. Antoniani, D.; Rossi, E.; Rinaldo, S.; Bocci, P.; Lolicato, M.; Paiardini, A.; Raffaelli, N.; Cutruzzolà, F.; Landini, P.; Appl. Microbiol. Biotechnol. 2013, 97, 7325.

28. Zhou, J.; Watt, S.; Wang, J.; Nakayama, S.; Sayre, D. A.; Lam, Y.; Lee, V. T.; Sintim, H. O.; Bioorg. Med. Chem. 2013, 21, 4396.

29. Spangler, C.; Kaever, V.; Seifert, R.; J. Pharmacol. Exp. Ther. 2011, 336, 234.

30. OpenEye Scientific Software, Santa Fe, NM. Available at http:// www.eyesopen.com, accessed in July 2017.

31. Knox, C.; Law, V.; Jewison, T.; Liu, P.; Ly, S.; Frolkis, A.; Pon, A.; Banco, K.; Mak, C.; Neveu, V.; Djoumbou, Y.; Eisner, R.; Guo, A. C.; Wishart, D. S.; Nucleic Acids Res. 2010, 39, D1035.

32. Ellingson, B. A.; Geballe, M. T.; Wlodek, S.; Bayly, C. I.; Skillman, A. G.; Nicholls, A.; J. Comput. Aided Mol. Des. 2014, 28, 289.

33. Tawa, G. J.; Baber, J. C.; Humblet, C.; J. Comput. Aided Mol. Des. 2009, 23, 853.

34. Wiggers, H. J.; Rocha, J. R.; Fernandes, W. B.; Sesti-Costa, R.; Carneiro, Z. A.; Cheleski, J.; da Silva, A. B. F.; Juliano, L.; Cezari, M. H. S.; Silva, J. S.; McKerrow, J. H.; Montanari, C. A.; PLoS Negl. Trop. Dis. 2013, 7, e2370.

35. Nicholls, A.; Grant, J. A.; J. Comput.-Aided. Mol. Des. 2005 , 19,661 .

36. Wiggers, H. J.; Rocha, J. R.; Cheleski, J.; Montanari, C. A.; Mol. Inf. 2011, 30, 565.

37. Hopkins, A. L.; Groom, C. R.; Alex, A.; Drug Discovery Today 2004, 9, 430.
38. Lamprecht, M.; Sabatini, D.; Carpenter, A.; Biotechniques 2007, 42,71 .

39. Chan, C.; Paul, R.; Samoray, D.; Amiot, N. C.; Giese, B.; Jenal, U.; Schirmer, T.; Proc. Natl. Acad. Sci. U. S. A. 2004, 101, 17084.

40. Zähringer, F.; Lacanna, E.; Jenal, U.; Schirmer, T.; Boehm, A.; Structure 2013, 21, 1149.

41. McGaughey, G. B.; Sheridan, R. P.; Bayly, C. I.; Culberson, J. C.; Kreatsoulas, C.; Lindsley, S.; Maiorov, V.; Truchon, J.-F.; Cornell, W. D.; J. Chem. Inf. Model. 2007, 47, 1504.

42. Hawkins, P. C. D.; Skillman, A. G.; Nicholls, A.; J. Med. Chem. 2007, 50, 74 .

43. Wheeler, T. J.; Clements, J.; Finn, R. D.; BMC Bioinf. 2014, 15,7 .

44. Kempen, E. C.; Brodbelt, J. S.; Anal. Chem. 2000, 72, 5411.

45. Siegal, G.; Ab, E.; Schultz, J.; Drug Discovery Today 2007, 12, 1032.

46. García-Raso, Á.; Fiol, J. J.; Rigo, S.; López-López, A.; Molins, E.; Espinosa, E.; Borrás, E.; Alzuet, G.; Borrás, J.; Castiñeiras, A.; Polyhedron 2000, 19, 991.

47. Elion, G. B.; J. Antimicrob. Chemother. 1983, 12, 9.

48. Voloshin, S. A.; Kaprelyants, A. S.; Biochemistry 2004, 69, 1268.

49. Massie, J. P.; Reynolds, E. L.; Koestler, B. J.; Cong, J.-P.; Agostoni, M.; Waters, C. M.; Proc. Natl. Acad. Sci. U. S. A. 2012, 109, 12746.

50. Henderson, G. B.; Huennekens, F. M.; Methods in Enzymology, vol. 122, $1^{\text {st }}$ ed.; Chytil, F.; McCormick, D., eds.; Elsevier: London, UK, 1986, p. 260.

51. Tsai, F. T. F.; Singh, O. M. P.; Skarzynski, T.; Wonacott, A. J.; Weston, S.; Tucker, A.; Pauptit, R. A.; Breeze, A. L.; Poyser, J. P.; O'Brien, R.; Ladbury, J. E.; Wigley, D. B.; Proteins: Struct., Funct., Genet. 1997, 28, 41.

52. Valle, J.; Solano, C.; García, B.; Toledo-Arana, A.; Lasa, I.; Trends Microbiol. 2013, 21, 364.

53. Fernicola, S.; Paiardini, A.; Giardina, G.; Rampioni, G.; Leoni, L.; Cutruzzolà, F.; Rinaldo, S.; J. Bacteriol. 2016, 198, 147.

54. Landini, P.; Antoniani, D.; Burgess, J. G.; Nijland, R.; Appl. Microbiol. Biotechnol. 2010, 86, 813.

55. Michel, M. C.; Foster, C.; Brunner, H. R.; Liu, L.; Pharmacol. Rev. 2013, 65, 809.

56. Reid, I. A.; Morris, B. J.; Ganong, W. F.; Annu. Rev. Physiol. 1978, 40, 377.

57. Nabah, Y. N. A.; Mateo, T.; Estellés, R.; Mata, M.; Zagorski, J.; Sarau, H.; Cortijo, J.; Morcillo, E. J.; Jose, P. J.; Sanz, M.-J.; Circulation 2004, 110, 3581.

58. Han, C.; Liu, J.; Liu, X.; Li, M.; Atherosclerosis 2010, 212, 206.

59. Raiden, S.; Nahmod, K.; Nahmod, V.; Semeniuk, G.; Pereira, Y.; Alvarez, C.; Giordano, M.; Geffner, J. R.; J. Pharmacol. Exp. Ther. 2002, 303, 45. 
60. Raiden, S.; Pereyra, Y.; Nahmod, V.; Alvarez, C.; Castello, L.; Giordano, M.; Geffner, J.; J. Leukocyte Biol. 2000, 68, 700.

61. Danilchanka, O.; Mekalanos, J. J.; Cell 2013, 154, 962.
62. Ma, D.-L.; Chan, D. S.-H.; Leung, C.-H.; Chem. Soc. Rev. 2013, $42,2130$.

Submitted: April 28, 2017 Published online: July 24, 2017 